\title{
Geometria Dinâmica 3D - novas perspectivas para o pensamento espacial
}

\author{
Márcia Notare - PPGEMAT/IME/UFRGS - marcia.notare @ gmail.com \\ Marcus Basso - PPGEMAT/IME/UFRGS - PPGIE - mbasso@ufrgs.br
}

\begin{abstract}
Resumo: Neste trabalho se discute possibilidades de contribuição do uso de ferramentas de Geometria Dinâmica 3D na aprendizagem de conceitos e na resolução de problemas de Geometria Espacial. A partir da resolução de problemas envolvendo construções geométricas tridimensionais e ancorados em conceitos piagetianos de representação do espaço, analisa-se as soluções propostas por estudantes de Curso de Pós-graduação via uso do software GeoGebra 3D. Os resultados apontam que o uso de software de geometria dinâmica amplia as possibilidades de raciocínio espacial.
\end{abstract}

Palavras-chave: GeoGebra, Geometria Dinâmica 3D, pensamento espacial, representação, resolução de problemas

\section{Dynamic Geometry 3D - new prospects for spatial thinking}

Abstract: This paper discusses possibilities of contribution of using Dynamic Geometry $3 \mathrm{D}$ tools in learning of concepts and in solving spatial geometry problems. From the resolution of problems involving three-dimensional geometric constructions and anchored in piagetian concepts of representation of space, we analyze the solutions proposed by students Postgraduate Course by use of GeoGebra 3D software. The results indicate that the use of dynamic geometry software expands the possibilities spatial reasoning.

Keywords: GeoGebra, Dynamic Geometry 3D, spatial thinking, representation, problem solving

\section{Introdução}

\begin{abstract}
"Quando se abrem os tradicionais livros de geometria do espaço quase, invariavelmente, encontra-se o mesmo receituário de fórmulas elaboradas que se fecham em si. Sub-repticiamente, apresenta-se este formulário como completo em si, como se nada mais existisse além dele. Mas a geometria é uma ciência que não conhece limites. Se eventualmente eles existirem, localizam-se na capacidade da imaginação humana". (WEIMER, 1985, p.145)
\end{abstract}

Percebe-se, mais recentemente, um tímido aumento do reconhecimento da importância da geometria para conceder uma boa formação matemática aos estudantes da Educação Básica (Gutierres, 2016). Esse reconhecimento é suportado pela disponibilidade de novas ferramentas de matemática dinâmica, como o GeoGebra, para apoiar a aprendizagem de Geometria. Esse apoio se manifesta de maneira bastante clara e consolidada através de artigos, teses e dissertações disponíveis em repositórios digitais em língua portuguesa e, ainda mais fortemente, em repositórios em língua inglesa. O próprio repositório do GeoGebra torna disponível centenas de objetos e propostas para uso do GeoGebra com estudantes de diferentes níveis de ensino. Porém, ainda são escassos os trabalhos acadêmicos sobre o uso da versão 3D do Geogebra nos processos de aprendizagem de Geometria Espacial.

Quando se aborda a Geometria Espacial, temos hoje à disposição ferramentas de geometria dinâmica 3D, que oferecem novas possibilidades para ensinar geometria espacial 
sem manipular sólidos espaciais em papel ou com outros materiais manipulativos. Assim, considerando a existência de uma multiplicidade de pesquisas (Hoyles, Lagrange, 2013; Shaffer e Clinton, 2006; Gravina, 2001; Santos, 2008) que apontam para a importância da Geometria Dinâmica (GD) na geometria plana, entende-se como necessário investigar seus impactos na aprendizagem de geometria espacial.

\section{A geometria dinâmica e o pensamento espacial}

A geometria dinâmica vem ganhando cada vez mais espaço nas investigações em Educação Matemática e tecnologias digitais (Sinclair, N \& Robutti, 2013; Lagrange, 2013; Moreno-Armella, Hegedus \&, Kaput, 2008). O desenvolvimento de sistemas dinâmicos voltados para a aprendizagem de Matemática tem possibilitado novas formas de tratamento para problemas, pela possibilidade de tornar acessíveis e manipuláveis objetos matemáticos que até então precisavam ser tratados de maneira estática e abstrata.

Uma das principais contribuições das tecnologias digitais para a educação matemática foi tornar possível a "concretização" dos objetos matemáticos na tela do computador, dando a sensação de realismo e de existência material pela possibilidade de manipulação e alteração de suas propriedades. Com a possibilidade de manipular os objetos matemáticos, surge uma nova forma de pensar e fazer matemática, configurando-se uma extensão do pensamento do indivíduo.

Shaffer e Clinton (2006), ao discutir sobre o papel das tecnologias digitais no pensamento humano, sugerem que, assim como as ferramentas são exteriorizações de projetos humanos, os pensamentos são internalizações de nossas ações com ferramentas. Para estes autores, todos os pensamentos estão ligados a ferramentas, e todas as ferramentas estão ligadas aos pensamentos, um não existindo sem o outro. Dessa forma, eles propõem que devemos tratar as ferramentas e os pensamentos com o mesmo status, ou seja, não há ferramentas sem pensar, e não há pensamento sem ferramentas. Existem apenas toolforthoughts (ferramentasparapensamento), que representam a relação recíproca entre ferramentas e pensamentos, ou entre pessoas e objetos. Shaffer e Clinton (2006) afirmam que, se utilizarmos as palavras ferramenta para o pensamento separadas, podemos estar sugerindo que o pensamento é a categoria mais ampla e que as ferramentas se constituem em algum artefato para ajudar as pessoas a pensar; ou podemos implicar que ferramenta é o quadro mais amplo e as pessoas são apenas agentes que usam pensamentos e artefatos físicos como ferramentas.

É desta forma que entendemos como as tecnologias digitais devem ser pensadas na atividade matemática. Em especial, acreditamos que a matemática dinâmica pode contribuir para o desenvolvimento do pensamento geométrico espacial, uma vez que, com os recursos disponíveis, torna-se possível concretizar sólidos espaciais não triviais de serem construídos e manipulados com objetos concretos físicos.

O raciocínio espacial consiste em um conjunto de processos cognitivos nos quais representações mentais, relações e transformações são construídas e manipuladas. A representação espacial constitui um sistema complexo de concepções, que vai além da percepção do indivíduo em geral. O espaço é subjetivo, é uma interpretação da realidade, e não necessariamente uma reprodução da realidade. Se, por um lado, a percepção é o conhecimento dos objetos resultante de um contato direto com eles, a representação consiste em completar o conhecimento perceptivo desses objetos referindo-se a outros não percebidos simultaneamente. Esse é o caso, por exemplo, da situação em que, "reconhecendo um triângulo, assimilamos a figura dada a toda classe das formas comparáveis não percebidas de maneira simultânea." (Piaget, 1993, p.32).

Mas a representação não constitui apenas um prolongamento da percepção. Ela, introduz um sistema de significações que contempla uma diferenciação entre o significante 
e o significado. O problema da passagem da percepção à representação espacial apoia-se simultaneamente no significante e no significado, isto é, na imagem e no pensamento. Quanto às relações intelectuais que constituem o espaço representativo elas são, em seu início, ligadas à imagem como a um suporte do qual dependem estreitamente, "mas à medida que atingem as transformações espaciais em oposição às figuras estáticas, dissociam o elemento motor próprio à imagem de seus elementos figurais e liberam-se destes últimos, a ponto de submetê-los a título de simples símbolos auxiliares." (Piaget, 1993, p.32)

Para Piaget (1993, p.474) a representação espacial

"é uma ação interiorizada e não simplesmente a imaginação de um dado exterior qualquer, resultado de uma ação. A representação espacial não chega, efetivamente, a prever esse resultado, e mesmo a reconstituir o de uma ação anterior, senão tornando-se ela mesma ativa, isto é, operando os objetos simbolizados como a ação opera sobre os objetos reais, ao invés de limitar-se a evocá-los."

Bishop (1983) sugeriu dois componentes espaciais que seriam especialmente relevantes para aprendizagem da matemática. O primeiro consiste na capacidade de interpretar a informação figural e envolve a compreensão de representações visuais. $O$ segundo refere-se à habilidade de processamento visual. Ela envolve a manipulação e a transformação das representações visuais e imagens, para além da tradução de relações presentes nas representações visuais observadas.

$\mathrm{Na}$ literatura existente, as pesquisas sugerem que um dos fatores determinante para a habilidade de raciocínio espacial é a capacidade de manutenção e manipulação de uma imagem com alta variedade de elementos e estímulos. Pessoas com habilidades espaciais bem desenvolvidas devem ser capazes de imaginar combinações espaciais de objetos a partir de diferentes pontos de vista e de manipular imagens visuais (Clements, 1992). Assim, o conceito de imagem desempenha um papel central no estudo das habilidades espaciais. Kosslyn (1983) define quatro classes de processo de imagem: gerar uma imagem, verificar e analisar uma imagem para responder questões sobre ela, transformar ou operar sobre uma imagem, e manter uma imagem a serviço de alguma operação mental. Dessa forma, os processos mentais que dão conta das habilidades espaciais devem ser capazes de conter informações que permitam a reconstrução da imagem, com um grau elevado de isomorfismo com a imagem externa observada.

Outro aspecto relevante é saber se a "abstração" das formas e outros elementos geométricos efetua-se a partir do objeto unicamente, ou a partir igualmente das ações que o sujeito aplica aos objetos (Basso, 1996). Ora, a abstração desses elementos não se dá segundo um processo análogo ao da abstração das qualidades físicas, como o peso ou a cor de um objeto. $\mathrm{Na}$ abstração desses elementos, por exemplo, a dimensão, há mais do que uma simples extração das qualidades inerentes ao objeto: há uma abstração em relação à ação, ou à coordenação das ações do sujeito. É o papel da coordenação de ações que confere a estes elementos um caráter geométrico e não somente físico. Essa coordenação de ações é que estará na base da construção do sistema de coordenadas 3D. De fato, um sistema de coordenadas de duas ou três dimensões, supõe, ao contrário da percepção, que se apoia em campos restritos, a coordenação operatória de todos os campos entre si. (Piaget, 1993)

A imagem que o sujeito extrai de um objeto é o que pode ser construído a partir de ações próprias realizadas sobre o objeto. Para formas complexas, a percepção pode falhar para realizar uma síntese e coordenação dos dados perceptivos que são baseadas em raciocínio. Para construir uma imagem mental é preciso antecipar possíveis características do objeto observado, como retas, curvas, ângulos, congruências, entre outros. 
Quanto à imagem, ela desempenha um papel de significante, ou de símbolo em relação a ação. "Papel importante, aliás, pois é na medida em que a ação material pode ser evocada por essa imitação interior constituída pela imagem que tal ação torna-se representativa." (Piaget, 1993, p.476). Mas não é a imagem que determina as significações, por mais fundamental que ela seja a título de suporte ou significante.

Hershkowitz (1998) afirma que, no pensamento, as pessoas não usam definições para conceitos, mas sim imagens conceituais, uma combinação de todas as imagens mentais e propriedades que têm sido associadas ao conceito.

A ação metal tem papel fundamental no processo de aprendizagem de geometria espacial e no desenvolvimento do pensamento geométrico espacial. Diversos estudos apontam para a importância de materiais manipulativos para apoiar a construção de imagens mentais. $\mathrm{O}$ uso de manipulações permite que os alunos possam experimentar suas ideias, analisar e refletir sobre elas, para modificá-las quando necessário. O uso apenas de desenhos para explorar a geometria espacial não é suficiente, pois desenhos estáticos no papel não representam objetos concretos e manipuláveis, ou seja, não é possível agir sobre o desenho de forma ampla e flexível, com o realismo necessário para apoiar a construção de imagens mentais adequadas. Nestas situações, acreditamos que o uso de construções em ambientes de geometria dinâmica pode auxiliar os alunos a construir imagens conceituais menos restritas.

Isso porque nem todos os problemas que envolvem habilidades espaciais são facilmente representados por materiais manipulativos concretos. Em situações assim, a utilização da geometria dinâmica pode proporcionar um espaço de construção e manipulação de situações mais complexas. É com este foco que apresentamos problemas cuja solução apoiou-se em construções dinâmicas.

\section{GeoGebra3D na resolução de problemas}

Nessa seção, apresentaremos e discutiremos dois problemas que envolvem o pensamento geométrico espacial, ambos aplicados em uma turma de Mestrado Profissional, cujo público são professores de Matemática da Educação Básica. Foi sugerido aos alunos que na solução dos problemas eles poderiam utilizar estratégias e recursos que considerassem adequados. Ou seja, o uso do GeoGebra3D para obter uma solução do problema, embora sugerido, não era obrigatório.

O GeoGebra3D é um recurso relativamente novo, disponibilizado em versão estável em 2014 e na qual professores e alunos, ainda em processo de elaboração do instrumento como recurso para pensar em matemática, estão desvendando e descobrindo suas ferramentas, suas potencialidades e suas fragilidades.

O primeiro problema apresentado está ilustrado na Figura 1.

Em um primeiro momento, alguns alunos buscaram resolver o problema apenas com lápis e papel, o que seria possível e viável. Contudo, observamos que diversos alunos construíram uma imagem figural equivocada dos sólidos destacados, levando à resposta errada de que todos seriam iguais.

A visualização da solução desse problema apenas com lápis e papel, de forma estática, proporciona uma manipulação restrita da situação. Para o desenvolvimento do pensamento geométrico espacial, é importante que as ações mentais estejam apoiadas em objetos manipuláveis, físicos ou virtuais, para construir imagens mentais adequadas que conduzam à solução do problema. Novamente, destaca-se que não é a imagem que determina essas significações. É a partir da coordenação das ações assimiladoras pelo sujeito que tais relações se estabelecem. 


\section{1. (ENEM 2011 - Questão 144)}

Uma indústria fabrica brindes promocionais em forma de pirâmide. A pirâmide é obtida a partir de quatro cortes em um sólido que tem a forma de um cubo. No esquema, estão indicados o sólido original e a pirâmide obtida a partir dele.
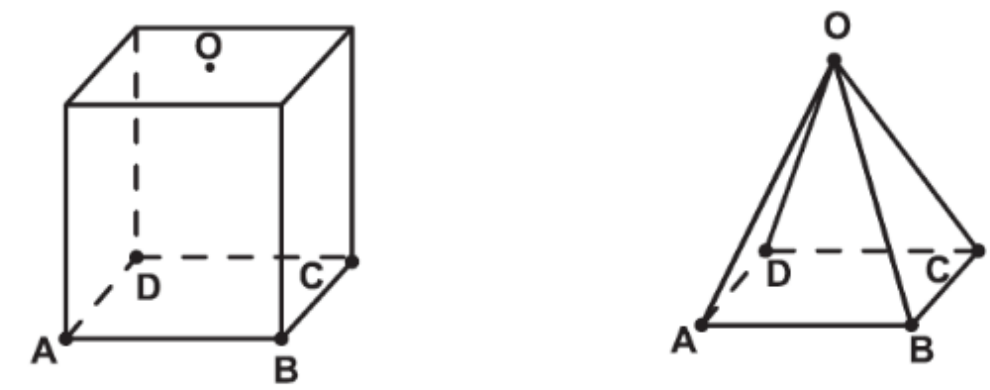

Os pontos A, B, C, D e O do cubo e da pirâmide são os mesmos. O ponto O é central na face superior do cubo. Os quatro cortes saem de $\mathrm{O}$ em direção às arestas $\overline{A D}, \overline{B C}, \overline{A B}$ e $\overline{C D}$, nessa ordem. Após os cortes, são descartados quatro sólidos

Os formatos dos sólidos descartados são
(A) todos iguais.
(B) todos diferentes.
(C) três iguais e um diferente.
(D) apenas dois iguais.
(E) iguais dois a dois.

Figura 1 - Questão do Exame Nacional do Ensino Médio, 2014

Depois de certo momento, incentivamos os alunos a representar a situação no GeoGebra3D, com todo o dinamismo e realismo que o programa proporciona. Para realizar a construção, foi preciso organizar as ideias, de modo a seguir passos precisos e ordenados: (1) construir o cubo inicial, que origina o problema; (2) construir os planos de corte do cubo, obedecendo a ordem em que são descritos pelo problema; (3) a partir dos planos construídos, para dar dinamismo e realismo à figura, construir os sólidos a serem destacados.

Observamos que os alunos ainda não haviam manipulado a versão $3 \mathrm{D}$ do GeoGebra. Dessa forma, simultaneamente à construção da solução do problema, os alunos exploraram seus menus e ferramentas, descobrindo as possibilidades que o software oferece, o que evidencia que o processo de apropriação do recurso pode ocorrer de forma concomitante com a aprendizagem de conceitos de matemática. Esse aspecto é revelador da contribuição para a aprendizagem em matemática gerada pelo uso e apropriação do próprio recurso digital de GD com os estudantes.

Para realizar a construção do dinamismo de destacar sólidos, os alunos utilizaram diferentes estratégias, como movimentos de rotação (aluno A) ou translação (aluno B), ou edição da coordenada de pontos cruciais no movimento (aluno A). As figuras 2 e 3 trazem duas soluções apresentadas pelos alunos A e B, respectivamente. A partir das construções realizadas, percebe-se que a solução do problema equivale a sólidos iguais dois a dois (dois prismas triangulares e duas pirâmides triangulares). Temos aqui um caso em que a geometria dinâmica permite a construção de imagens mentais isomorfas à situação real, dando suporte para o pensamento geométrico espacial. Como referido na seção 2, a coordenação de ações é que dará o suporte para que estes objetos tenham um caráter geométrico e não apenas físico. 


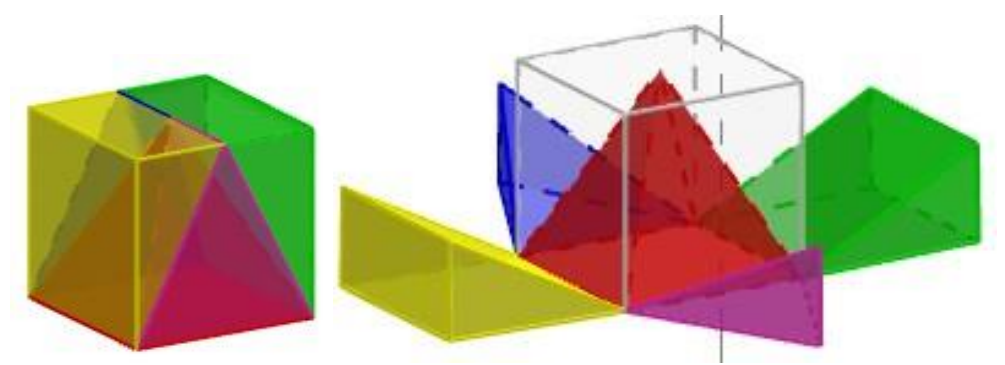

Figura 2 - Solução do estudante A, com movimento de rotação
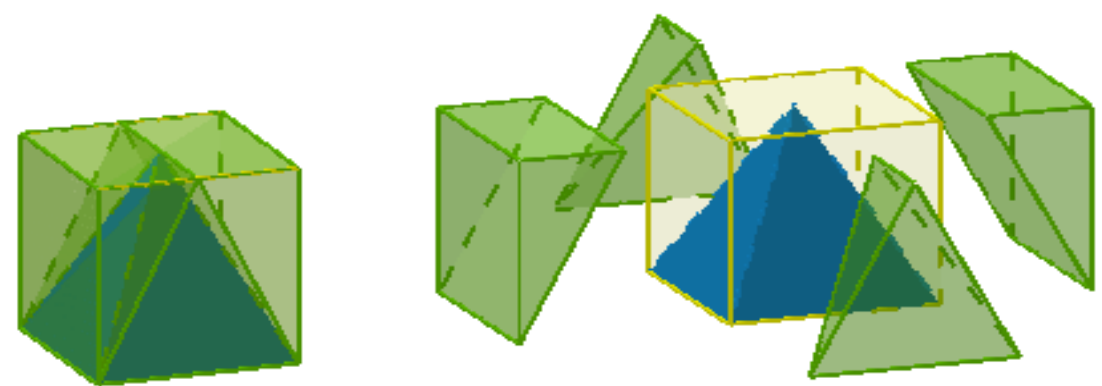

Figura 3 - Solução do estudante B, com movimento de translação

Analisando a construção de $\mathrm{A}$, percebemos que utilizou um controle deslizante para criar uma variável $a$, com variação entre $0^{\circ}$ e $90^{\circ}$, que foi posteriormente utilizada na edição das coordenadas de pontos dos sólidos que estariam em movimento, conforme ilustram as Figuras 4 e 5.

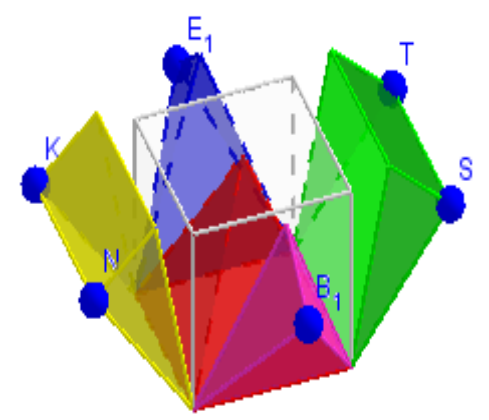

Figura 4 - Solução do estudante A, com edição das coordenadas de pontos

\begin{tabular}{|c|c|c|c|}
\hline Redefinir & & & $x$ \\
\hline \multicolumn{4}{|l|}{ Ponto K } \\
\hline$(-2 \cos (a), 2,2 \operatorname{sen}(a)) \mid$ & & & a \\
\hline Propriedades... & OK & Cancelar & Aplicar \\
\hline 82 Redefinir & & & $x$ \\
\hline \multicolumn{4}{|l|}{ Ponto T } \\
\hline$(2+2 \cos (a), 2,2 \operatorname{sen}(a))$ & & & a \\
\hline Propriedades... & OK & Cancelar & Aplicar \\
\hline 52 Redefinir & & & $x$ \\
\hline \multicolumn{4}{|l|}{ Ponto E_1 } \\
\hline$(1,2+2 \cos (a), 2 \operatorname{sen}(a))$ & & & a \\
\hline Propriedades... & OK & Cancelar & Aplicar \\
\hline
\end{tabular}

\begin{tabular}{|c|c|c|c|}
\hline 28 Redefinir & & & $x$ \\
\hline \multicolumn{4}{|l|}{ Ponto N } \\
\hline$(-2 \cos (a), 0,2 \operatorname{sen}(a))$ & & & a \\
\hline Propriedades... & OK & Cancelar & Aplicar \\
\hline Gedefinir & & & $x$ \\
\hline \multicolumn{4}{|l|}{ Ponto S } \\
\hline$(2+2 \cos (a), 0,2 \operatorname{sen}(a)$ & & & a \\
\hline Propriedades... & OK & Cancelar & Aplicar \\
\hline 3 Redefinir & & & $x$ \\
\hline \multicolumn{4}{|l|}{ Ponto B_1 } \\
\hline$(1,-2 \cos (a), 2 \operatorname{sen}(a))$ & & & a \\
\hline Propriedades... & OK & Cancelar & Aplicar \\
\hline
\end{tabular}

Figura 5 - Estratégia de construção do estudante A - janelas de edição de coordenadas de pontos 
Já o aluno B construiu, no plano da base do cubo, quatro vetores sobre os eixos coordenados $x$ e $y$, de comprimento livre, que dariam o movimento de translação dos sólidos a serem destacados, como ilustra a Figura 6.
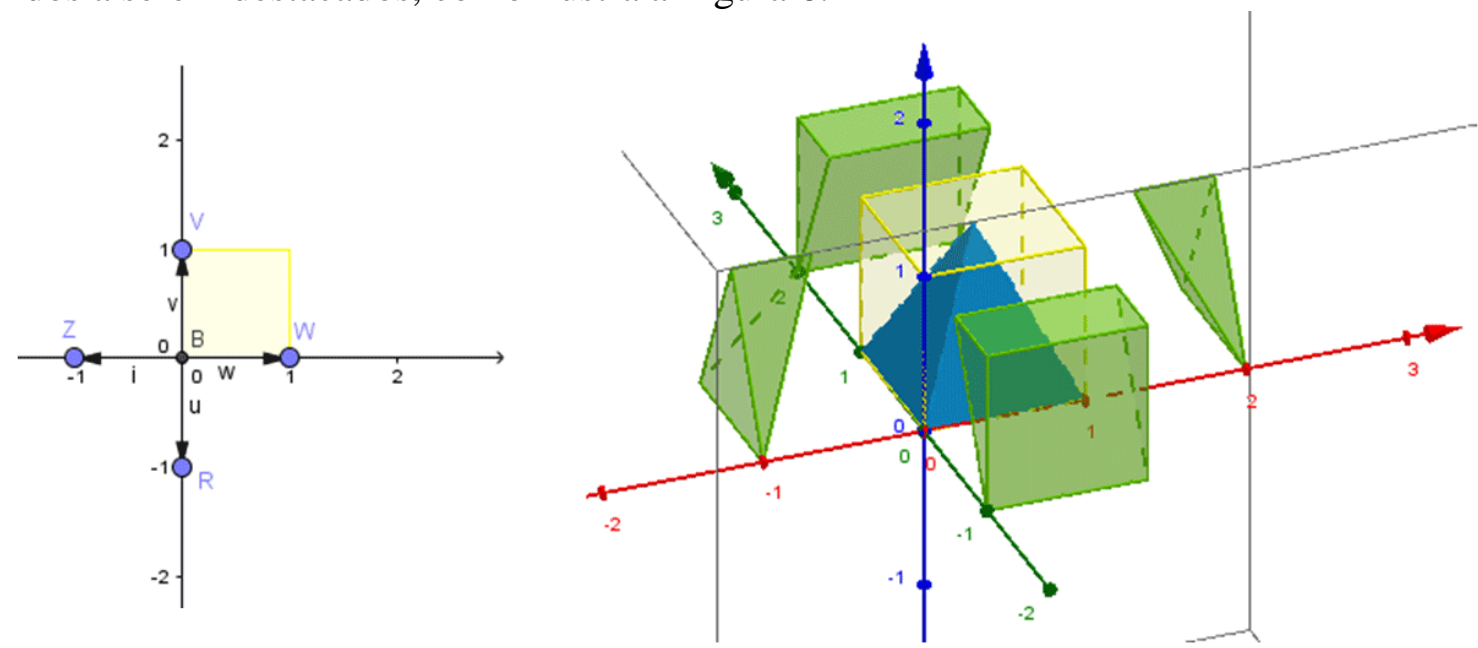

Figura 6 - Estratégia de construção do estudante B

Observa-se que a solução apresentada por B, pressupõe a construção de um sistema de coordenadas tridimensional, o que somente é possível a partir da abstração de conceitos como dimensão, orientação, multiplicação cartesiana, os quais, sem dúvida, não constituem qualidades inerentes ao objeto. Ou seja, há uma abstração em relação à ação, ou à coordenação das ações do sujeito. Novamente, será a coordenação de ações que conferirá a tais conceitos e objetos seu caráter matemático e não somente físico.

O segundo problema, baseado no trabalho de Lieban (2012), está apresentado na Figura 7.

A superfície lateral de uma vela com a forma cilíndrica está envolvida em papel. Essa foi seccionada de acordo com a figura abaixo e esse corte produziu dois sólidos. Retirando o papel de cada sólido e fazendo um corte no sentido horizontal (ver figura abaixo) obtém-se duas planificações. Represente no plano cada uma dessas planificações.
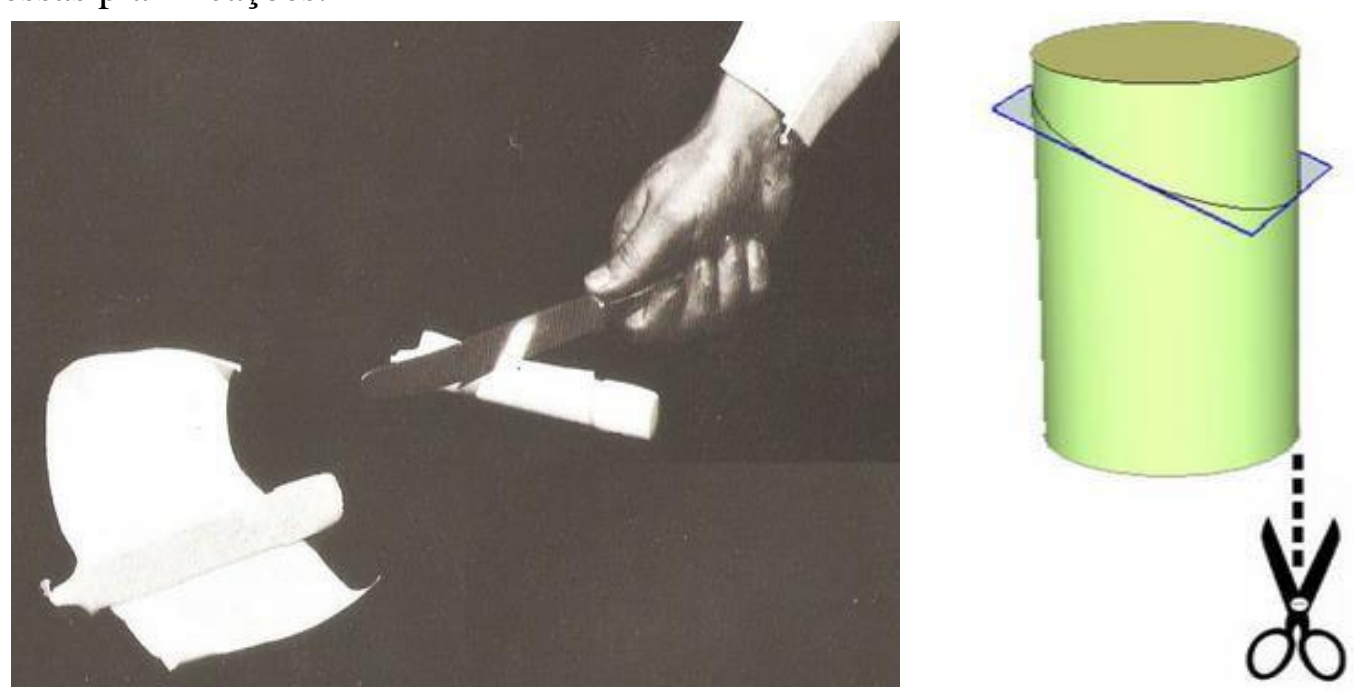

Figura 7 - Segunda questão apresentada 
Iniciar a discussão deste problema com a manipulação de objetos físicos pode levar a uma discussão interessante, mas não necessariamente à solução correta do problema.

Neste problema, apoiar-se sobre a percepção da imagem figural pode não ser suficiente para evocar a figura planificada, dada a complexidade do problema ao envolver dados que precisam ser antecipados por não estarem presentes na figura espacial representada em desenho. Para construir a imagem mental da planificação do papel que envolve a vela, torna-se importante apoiar-se sobre a manipulação do objeto, agir sobre ele, para construir imagens com o realismo necessário que conduza à solução do problema. Temos aqui um exemplo concreto no qual o ambiente de geometria dinâmica 3D é necessário para apoiar o pensamento geométrico espacial.

Para resolver o problema, os alunos partiram diretamente para a construção de um cilindro e um plano secante no GeoGebra3D. Observamos os alunos manipulando o plano secante e o cilindro, mas a construção realizada não era suficiente para construir a imagem mental das planificações. Alguns alunos apresentaram soluções como a ilustrada na Figura 8.
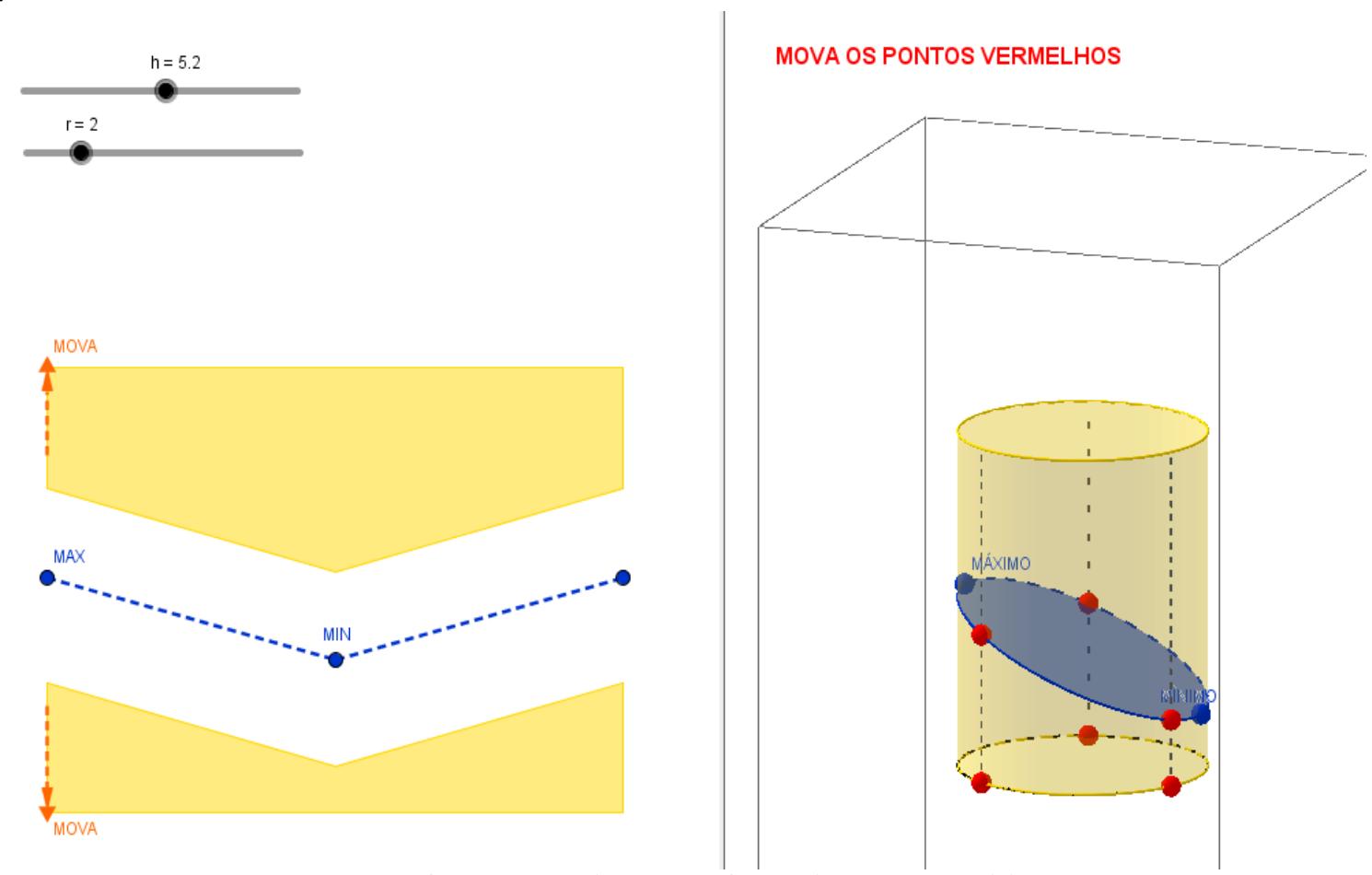

Figura 8 - Solução equivocada para o problema

Para resolver o problema, é preciso construir dinamicamente a planificação da folha, tendo como suporte a ideia de área como preenchimento de superfície, pois o software não gera a planificação desta construção automaticamente. Assim, os alunos que chegaram à solução do problema, entenderam que deveriam construir um ponto que se move sobre a circunferência da base do cilindro e um ponto gerado pela intersecção da reta perpendicular ao plano da base do cilindro com o plano que secciona o cilindro. Estes dois pontos determinam um segmento que percorre a superfície lateral de uma das partes recortadas do cilindro. O recurso de rastro, disponível no software, para esse segmento oferece a imagem da planificação, como ilustra a Figura 9. A região preta, traçada no plano xOy, determinada pelo rastro do segmento, representa a planificação da parte inferior do papel que envolve o cilindro. 


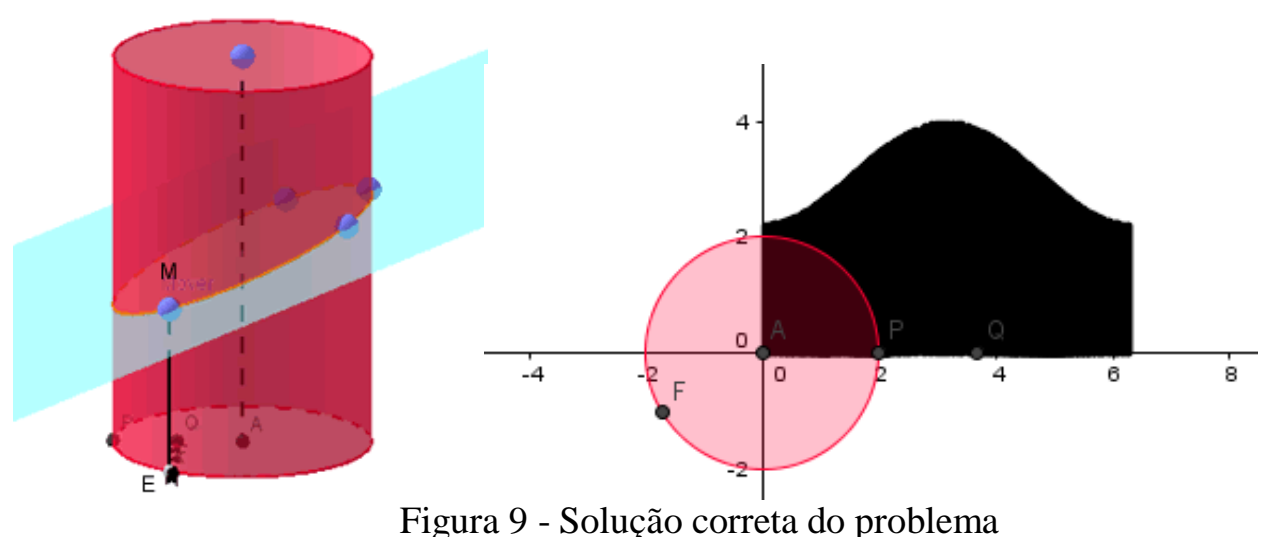

Embora não seja evidente, a percepção, no contexto da solução desse problema é enganosa, pois os elementos perceptivos não são suficientes para levar o aluno a representar mentalmente a curva produzida pelo corte no cilindro. Ao contrário, pelo fato da seção resultante do corte no cilindro ser plana, sugere que a curvatura da planificação é retilínea. Ora, não há relação entre o fato dessa seção ser plana e o corte ser retilíneo. De fato, o corte será uma curva cujo comportamento somente poderá ser interpretado corretamente pelo estudante a partir da coordenação de ações envolvendo representações espaciais entre o movimento do ponto $\mathrm{E}$ que percorre a circunferência da base e o ponto $\mathrm{M}$ que percorre a elipse da seção plana gerada pelo corte no cilindro. Essa coordenação de ações origina-se do conceito de superfície, o qual é essencial para estabelecer uma estratégia adequada de solução para o problema.

\section{Considerações finais}

O uso de recursos de geometria dinâmica 3D pode gerar altos níveis de pensamento geométrico, contribuindo para o desenvolvimento do raciocínio espacial dos estudantes. Essa afirmação encontra suporte no conjunto de dados resultantes das soluções apresentadas pelos estudantes desse estudo e na teoria piagetiana sobre a representação do espaço.

O ambiente de geometria dinâmica amplia as possibilidades de estabelecerem coordenações de ações que conduzem os estudantes a criarem relações corretas do ponto de vista matemático. Isso deve-se ao fato do ambiente oferecer um suporte que permite manipulações e transformações geométricas enriquecedoras do pensamento espacial, o que, por sua vez, torna-se uma base importante para a compreensão de conceitos geométricos abstratos.

\section{Referências}

BASSO, M. V. A. Representação do Espaço - Um Estudo de Processos Psicológicos Subjacentes ao Trabalho com Objetos 2D e 3D através de Mapeamento Cerebral e da Teoria Psicogenética. (Dissertação de Mestrado). Pós Graduação em Psicologia do Desenvolvimento, Universidade Federal do Rio Grande do Sul, Porto Alegre, 1996.

BISHOP, A.J. Space and geometry. Em LESH, R. and Landau, M. (Eds.), Acquisition of Mathematics Concepts and Processes (pp. 175-203). New York: Academic Press, 1983.

CLEMENTS, D. H., e BATTISTA, M. T. Geometry and spatial reasoning. Em D. A. Grouws (Ed.), Handbook of research on mathematics teaching and learning (pp. 420464). New York: Macmillan, 1992. 
GOLDENBERG, P. Thinking (And Talking) About Technology in Math Classrooms. In: Education Development Center, 2000. Disponível em: http://www2.edc.org/mcc/PDF/iss_tech.pdf. Acesso: 30 set. 2016.

GRAVINA, Maria Alice. Os ambientes de Geometria Dinâmica e o Pensamento Hipotético-Dedutivo. Tese (Doutorado) - Programa de Pós-Graduação em Informática na Educação - Universidade Federal do Rio Grande do Sul, Porto Alegre, 2001. Disponível em http://www.lume.ufrgs.br/handle/10183/2545. Acesso: 30 set. 2016.

HERSHKOWITZ, R. Reasoning in Geometry. Em HERSHKOWITZ, R, DUVAL, R., BUSSI, M.G., BOERO, P. LEHER, R. ROMBERG, T. BERTHELOT, R., SAIN, M.H., SALIN, K. J. Perspectives on the Teaching of Geometry for the 21st Century. Volume 5 of the series New ICMI Study Series (pp 29-83), 1998.

HOYLES, C., LAGRANGE, J. B. (Eds.) Mathematics Education and TechnologyRethinking the Terrain. The 17th ICMI Study. Londres: Springer, 2010.

KOSSLYN, S. M. Ghosts in the mind's machine. New York: W. W. Norton, 1983.

LAGRANGE, J.B. Les technologies numériques pour lénseignement: usages, dispositifs et genèses. Toulouse: OCTARÈS Editions, 2013.

LIEBAN, D. E. Palmitos \& Da Vinci: do concreto ao digital. Inspirações para movimentos articulados e parametrização de curvas com o GeoGebra. Oficinas - VI Bienal da Sociedade Brasileira de Matemática. Unicamp, 2012. Disponível em http://bienaldematematica.org.br/sites/bienaldematematica.org.br/files/Oficinas_1.pdf Acesso: 20 maio 2016.

MORENO-ARMELLA, L. HEGEDUS, S. J., KAPUT, J.J. From static to dynamic mathematics: historical and representational perspectives. Educational Studies in Mathematics. v.68, p 99-111, 2008.

MORENO-ARMELLA, L. Mathematical thinking and technology: some views on their coevolution. In: TSG15-ICME-10, Copenhagen. Disponível em http://matrix.skku.ac.kr/sglee/album/TSG 15_The role and use of technology in the teaching and learning of mathematics.pdf. Acesso: 21 set 2016.

PIAGET, J. e INHELDER, B. A Representação do Espaço na Criança. Artes Médicas: Porto Alegre, 1993.

SHAFFER, W. D.; CLINTON A. K. Toolforthoughts: Reexamining Thinking in the Digital Age. Mind, Culture and Activity, vol. 13, n. 4, California, 2006.

SINCLAIR, N and ROBUTTI, O. Technology and the Role of Proof: The Case of Dynamic Geometry. In: CLEMENTS, M.A., BISHOP, A., KEITEL, C, KILPATRICK, J., LEUNG, F. (Eds.). Third International Handbook of Mathematics Education, New York:

Springer, 2013, p. 571-596.

WEIMER, G. Empacotamento Fechado de Poliedros. Porto Alegre: Editora Sulina, 1985. 